\section{ATU-10 QUPATH MACHINE LEARNING ALGORITHM ACCURATELY IDENTIFIES MLH1 DEFICIENT INFLAMMATORY BOWEL DISEASE-ASSOCIATED COLORECTAL CANCER}

\begin{abstract}
1,2Ross Porter, ${ }^{3}$ Shahida Din, ${ }^{4}$ Peter Bankhead, ${ }^{1}$ Anca Oniscu, 'Mark J Arends. 'Division of Pathology, Centre for Comparative Pathology, Edinburgh Cancer Research Centre, Institute of Genetics and Molecular Medicine, Western General Hospital, University of Edinburgh, Scotland UK; ${ }^{2}$ Centre for Inflammation Research, Queen's Medical Research Institute, University of Edinburgh, Scotland UK; ${ }^{3}$ Edinburgh IBD Unit, Western General Hospital, NHS Lothian, Scotland UK; ${ }^{4}$ Centre for Genomic and Experimental Medicine, Institute of Genetics and Molecular Medicine, Western General Hospital, University of Edinburgh, Scotland UK
\end{abstract}

10.1136/gutjnl-2021-BSG.43

Introduction Mismatch Repair (MMR) deficient colorectal cancers are an important cohort to study as these patients have a high neo-epitope load, suggesting they may respond well to immunotherapy. We previously reported deficient MMR due to loss of MLH1 expression occurs in $>20 \%$ of all inflammatory bowel disease-associated colorectal cancers (IBD-CRC). Manually identifying MHL1 deficient tumours from immunostained samples is time-consuming and labour-intensive. Therefore, we aimed to train QuPath, a digital image analysis program, to accurately identify MLH1 deficient IBD-CRCs from a tissue microarray (TMA) containing normal colon and IBD-CRC.

Methods A TMA ( $\mathrm{n}=162$ cores) containing normal colon and IBD-CRC was immunostained for MLH1, digitalised and imported into QuPath. A representative sample (14 sections of $500 \mu \mathrm{m} \times 500 \mu \mathrm{m}$ ) was used to train a QuPath algorithm to identify tissue histology (normal epithelium, tumour, immune infiltrate or stroma) and MLH1 expression pattern. The algorithm was applied to the whole TMA. Accuracy level for histology and MLH1 classification was set at $>75 \%$ of cells per category; cores where neither category met this threshold were flagged for review and these cores were histopathologically reviewed qualitatively. Data were exported to IBM $^{\circledR}$ SPSS ${ }^{\circledR}(\mathrm{V} 25.0)$ for statistical analysis.

Results Tissue histology and MLH1 expression was correctly identified in $87 / 113(77 \%)$ cases, misclassified in $1 / 113$ $(0.9 \%)$ case and flagged for manual review in $25 / 113$ $(22.1 \%)$ cases. Reasons cores were flagged for review included: small quantity of tissue, atypical morphology, excessive immune infiltrate, normal epithelium and patchy immunostaining. Of cores not flagged for review $(n=88)$, the algorithm was highly sensitive (100\%) and specific (98.6\%) for identifying MLH1-deficient IBD-CRC, $\square=0.967$ (95\%CI $0.902-1.032)(\mathrm{p}<0.001)$

Conclusions MLH1 deficient IBD-CRC can be accurately identified using our QuPath algorithm, with an acceptable proportion of equivocal samples highlighted for manual evaluation. This process could be efficiently automated in conventional NHS IBD-CRC surveillance and treatment programmes to examine all colonic tissue and tumour specimens for MLH1 expression, thus identifying patients who may respond well to immunotherapy. This approach now needs to be validated using endoscopic biopsies.
Liver

\section{ATU-7 INCIDENT ACUTE KIDNEY INJURY HAS A WORSE PROGNOSIS THAN BASELINE IN SEVERE ALCOHOLIC HEPATITIS}

${ }^{1}$ Luke Tyson*, ${ }^{1}$ Professor Mark Thursz, ${ }^{2}$ Ewan Forrest, ${ }^{3}$ Michael Allison, ${ }^{1}$ Nikhil Vergis, ${ }^{1}$ Stephen Atkinson. 'Imperial College London, London, UK; ${ }^{2}$ NHS Greater Glasgow and Clyde, Glasgow, UK; ${ }^{3}$ Cambridge University Hospitals NHS Foundation Trust, Cambridge, UK

\subsection{6/gutjnl-2021-BSG.44}

Introduction Alcoholic hepatitis (AH) is the most severe alcohol-related liver disease. Acute kidney injury (AKI) is associated with increased mortality. AKI may be present at the time of presentation (baseline) or develop subsequently (incident). We used data from the STOPAH (STeroids Or Pentoxifyline for Alcoholic Hepatitis) trial to describe the prevalence of AKI, its association with mortality and risk factors for its development.

Methods The primary endpoint in analysis was 90-day mortality. Patients from the STOPAH trial were classified as having a baseline or incident AKI (within 7 days of starting treatment; D7). AKI was defined as any of: i) creatinine $\geq 26.5$ micromol/L above or $\geq 1.5 \mathrm{x}$ the lowest recorded creatinine; ii) creatinine $\geq 133 \mathrm{micromol} / \mathrm{L}$; iii) renal replacement therapy. The effect of AKI on 90-day mortality was tested by Kaplan-Meier survival analysis. Factors associated with incident AKI were compared by Student's t-test, Mann-Whitney U test or Chi-squared test as appropriate.

Results Baseline creatinine was recorded in 1051 patients; 282 patients with a normal creatinine at baseline were alive at D7 but did not have a second creatinine recorded so were excluded from survival analysis. Baseline AKI was present in 198/1051 (19\%) patients while 119/1051 (11\%) developed an

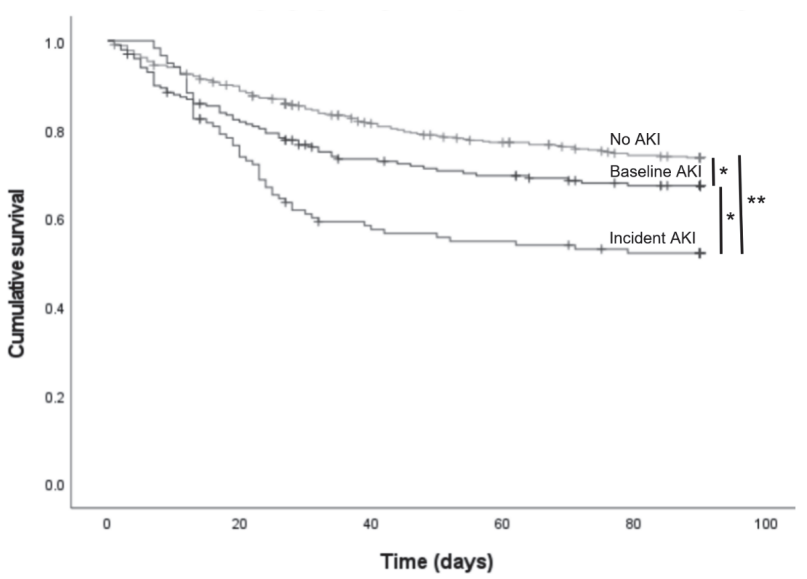

Abstract ATU-7 Figure 1 Kaplan-Meier survival curves for patients with a baseline acute kidney injury ( $n=198,63$ events), incident acute kidney injury ( $n=119,56$ events) and normal baseline and day seven creatinine ( $n=452,115$ events)

*Beslow (Generalised Wilcoxon) $p<0.05 ;{ }^{* *} p<0.001$ 